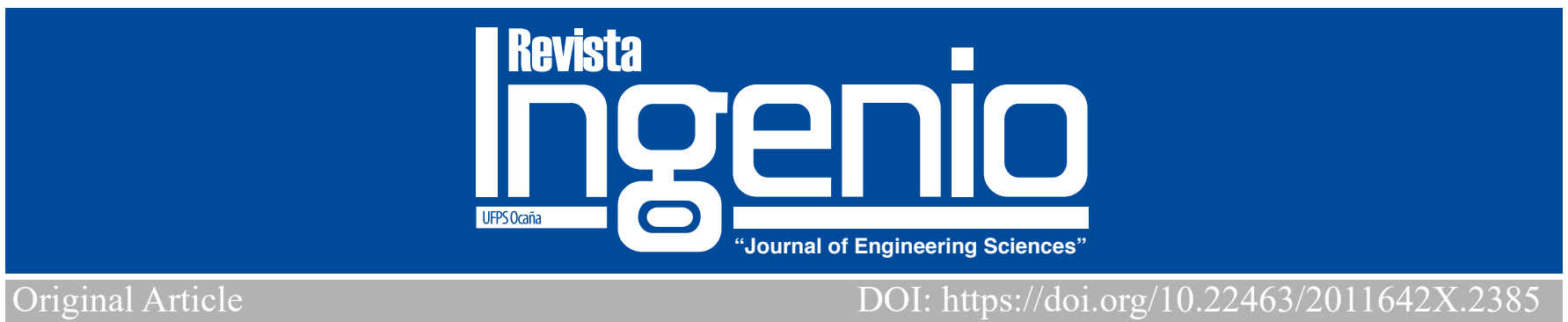

\title{
Diseño geométrico de una zapata doble para cimentación aplicada en torres de transmisión eléctrica
}

Double footing geometry design for the foundation applied in transmission towers

$$
\text { Ing. Mauricio Pérez Giraldo }{ }^{1} \text {, Est. Jose David Yepes Tumay }{ }^{2}
$$

${ }^{1}$ Instituto tecnológico metropolitano, Medellin, Colombia, https://orcid.org/0000-0002-6217-6859, Email: Maperezgi@unal.edu.co

${ }^{2}$ Universidad Nacional de Colombia- Sede Medellin, Colombia, https://orcid.org/0000-0002-6570-3546, Email: Jdyepest@unal.edu.co

Como citar: M. Pérez \& J. D. Yepes, "Diseño geométrico de una Zapata doble para cimentación aplicada en torres de transmisión elétrica", Revista Ingenio, vol. 18 (1), pp.17-24, 2021

\section{RESUMEN}

Palabras claves:

Cimentaciones, diseño computacional, eléctrica, geometría, torres, zapatas, FEM.
En el mundo actual las torres de transmisión eléctrica tienen un sistema de cimentación cuya geometría se asemeja a una zapata; este diseño ha presentado grandes ventajas y se le han dado diferentes enfoques de estudio, desde la forma en que se construye, el sitio de construcción y si se puede realizar de forma modular; el enfoque de investigación será el rediseño de esta geometría planteando un cambio a la cual denominaremos geometría de zapata doble. Este diseño será probado de forma computacional a través de software de asistencia ingenieril, CAE (computer aided engineering). El análisis muestra que la geometría planteada soporta las cargas a las que una cimentación normalmente está sometida validando que es un sistema posible para su implementación y sustitución de la geometría actual. Se destaca que para cumplir con las condiciones de operación el volumen de material de construcción es menor con respecto a la geometría actual, alivianando los costos de la cimentación de cada torre, teniendo un menor valor de construcción, presentando así una ventaja con respecto a la cimentación de zapata simple.

\begin{tabular}{ll}
\hline ABSTRACT \\
\hline Keywords: & $\begin{array}{l}\text { In actual world, electric transmission towers have a foundation system whose geometry resembles a footing; This design has presented } \\
\text { great advantages and has been given different study approaches, from the way it is built, the construction site and if it can be done } \\
\text { in a modular way; The research focus will be the redesign of this geometry introducing a change which we will call double footing }\end{array}$ \\
$\begin{array}{l}\text { Foundations, } \\
\text { computational }\end{array}$ & $\begin{array}{l}\text { geometry. This design will be tested computationally through CAE (computer aided engineering) software. The analysis shows that } \\
\text { the proposed geometry supports the loads to which a foundation is normally subjected, validating that it is a possible system for its } \\
\text { design,electric, } \\
\text { towers,footing }\end{array}$ \\
implementation and replacement of the current geometry. It is highlighted that in order to comply with the operating conditions, the \\
volume of construction material is less compared to the current geometry, alleviating the costs of the foundation of each tower, having \\
a lower construction value, thus presenting an advantage with respect to the simple footing foundation.
\end{tabular}

\section{Introducción}

La expansión tecnológica avanza de manera acelerada facilitando y mejorando los niveles de calidad de vida de las personas. No obstante, estos avances encuentran limitaciones para llegar a nuevas poblaciones, de origen rural generalmente, donde muchas de las cuales no han tenido mayor interacción con estos avances principalmente por dos factores, el costo y las limitaciones de ubicación. Aunque el mercado ha permitido el acceso a dispositivos eléctricos y electrónicos mediante líneas económicas que los hace accesibles, sigue prevaleciendo el problema de la locación, no solo por el traslado de productos grandes si no por el requerimiento de conexión eléctrica para su funcionamiento.
Este factor ha limitado el uso de estas herramientas por parte de dichas poblaciones rurales y se ha convertido en un reto a nivel de ingeniería y sociedad, puesto que, si bien se debe brindar el servicio de energía eléctrica estable y confiable, los altos costos que implica construir una línea de transmisión eléctrica que supla este objetivo, lo hacen poco atractivo para las diferentes empresas del sector.

Cada torre de transmisión tiene unos costos muy elevados, lo cual desincentiva su construcción y deja zonas sin acceso a energía eléctrica por ende obligadas al uso de plantas de generación a partir de combustibles fósiles que generan altas emisiones contaminantes. Dentro de los costos 
asociados al proceso de conexión uno de los más grandes es la construcción de las torres, principalmente de las fundaciones o cimentaciones de estas, las cuales requieren de excavación, $\mathrm{y}$ transporte de gran cantidad de material.

Estas cimentaciones son las encargadas de soportar las cargas asociadas a la torre y de asegurar la misma para que cumpla su función durante una vida útil superior a 50 años de servicio.

Actualmente hay diferentes líneas de investigación sobre las cimentaciones, con la intención de reducir su costo. Se observan aditivos de nutrientes tecnológicos para mejorar las características mecánicas de los materiales [1], mejoras del suelo de construcción [2], mejoras de la resistencia de los materiales a través de nanoaditivos como el nanosílice, mejorando la resistencia a la compresión [3], disminución del tiempo de fraguado [4] y construcción modular [5].

En la actualidad el sistema más utilizado para las diferentes geometrías de las torres de transmisión [6] es la construcción en sitio de cimentaciones con geometría de zapatas; usualmente piramidales o planas dependiendo del suelo y las cargas a las que está sometida la torre [7], las cuales requieren de grandes excavaciones y volúmenes de material, debido a la rigidez que estas tienen [8]. A su vez el tiempo de construcción es demasiado largo, debido a que se requiere un tiempo de fraguado del hormigón para llegar a unas condiciones óptimas de trabajo.

Debido a esto se hace necesario buscar alternativas que permitan una reducción de costos en la construcción de forma que sea viable y atractivo para las empresas realizar proyectos de conexión hacia estas zonas. En el presente trabajo se plantea el cambio de la estructura de cimentación de zapata convencional (la cual para el presente trabajo se denominará zapata simple) por una geometría óptima y que logre distribuir de forma más uniforme las cargas a las que es sometida, ya sea de tensión o de hundimiento. Se llegó a que una de las posibles opciones de diseño sería la implementación de otra zapata, que logre darle mayor estabilidad debido a que esta tiene más área de contacto con el terreno por lo cual logra transmitir mejor las cargas provenientes de la torre de transmisión, generando una estructura más sólida y resistente, a su vez requiriendo menor cantidad de materiales y volumen de excavación conllevando a ahorros en su construcción. Para el presente trabajo esta geometría se llamará cimentación de zapata doble.

Se propone para estudios futuros el uso de módulos prefabricados para reducir los factores de costo antes mencionados, como los propuestos para la cimentación de viviendas que en la actualidad se usan [9], estos módulos también podrían reducir el tiempo de fraguado el cual según la literatura se aproxima a los 28 días [10].
Para ello se realizó un análisis estático de la torre en condiciones críticas, dadas por la carga de la estructura y cargas de viento [11]. Esto se realiza con ayuda de un software de simulación, usando el método de elementos finitos (FEM por sus siglas en inglés).

\section{Desarrollo y metodología}

Se plantea el concepto de un sistema de cimentación de zapata doble, el cual se basa en una reestructuración de la cimentación convencional de zapata simple (Figura 1), con el añadido de una segunda zapata, la cual está ubicada encima de la zapata inferior (Figura 2). Esto bajo la hipótesis de que la distribución de cargas se realiza en las dos zapatas, lo cual provoca una disminución en las dimensiones finales y por tanto en el volumen final de construcción en la cimentación.

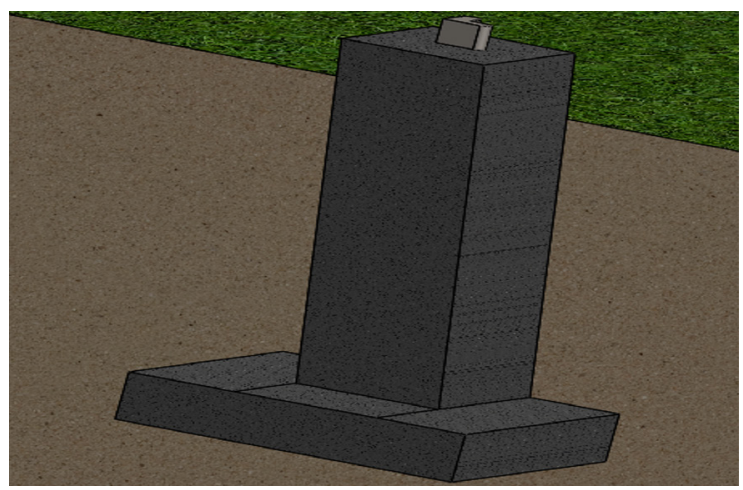

Figura 1. Cimentación de zapata simple

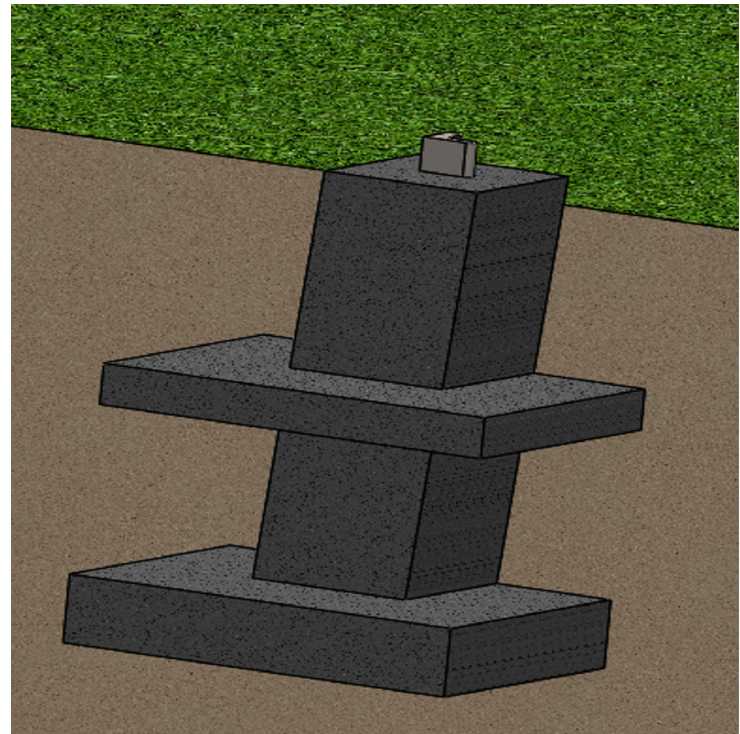

Figura 2. Cimentación de zapata doble 
Para el análisis se tomó en cuenta las cargas verticales y transversales generadas por los cables de transmisión y el cable de tierra dadas por la ecuación 1 y ecuación 2 [11].

$$
\begin{aligned}
& V=P l c \cdot D p b+\text { Pact } \\
& T=W P\left(\frac{\varphi C a b l e}{12}\right) D p+0.0872 \cdot(T i)
\end{aligned}
$$

Donde Plc se refiere el peso lineal del cable, Dpb es la distancia más baja entre puntos de la línea antes y después de la línea de transmisión, Pact se refiere al peso del aislador, WP hace referencia a la presión del viento, $\varphi$ cable al diámetro del cable, Dp es el promedio de distancia entre las torres vecinas, siendo usada para calcular las cargas de viento del cable y $\mathrm{T}_{i}$ la tensión inicial del cable.

De igual forma las cargas producidas por los vientos en las diferentes zonas de la estructura fueron tomadas en cuenta, calculadas por la ecuación 3 , donde $W_{i}$ corresponde a las cargas de viento sobre la estructura en dirección perpendicular a la estructura, $C f_{i}$ se refiere al coeficiente de fuerza para la zona estudiada y $\mathrm{A}_{i}$ al área donde se proyecta el viento [11].

$$
W_{i}=W P \cdot C f_{i} \cdot A_{i}
$$

Para la validación de la geometría se utiliza el software ANSYS 20 R2, con el objetivo de realizar el análisis FEM; se usan elementos tetraédricos usando una combinación de orden de los elementos, dado que la región del hormigón tiene geometría mucho mas cuadrada, mientras que la chimenea tiene mayores curvas, por esto se usarán elementos tanto lineales como cuadráticos. La geometría de estudio considerada será la correspondiente a la de zapata doble antes mencionada, tener en cuenta que tanto la geometría de zapata doble como la de zapata simple son cuadradas. En la figura 3 se observan las dimensiones de la zapata doble (unidades en $\mathrm{mm}$ ).

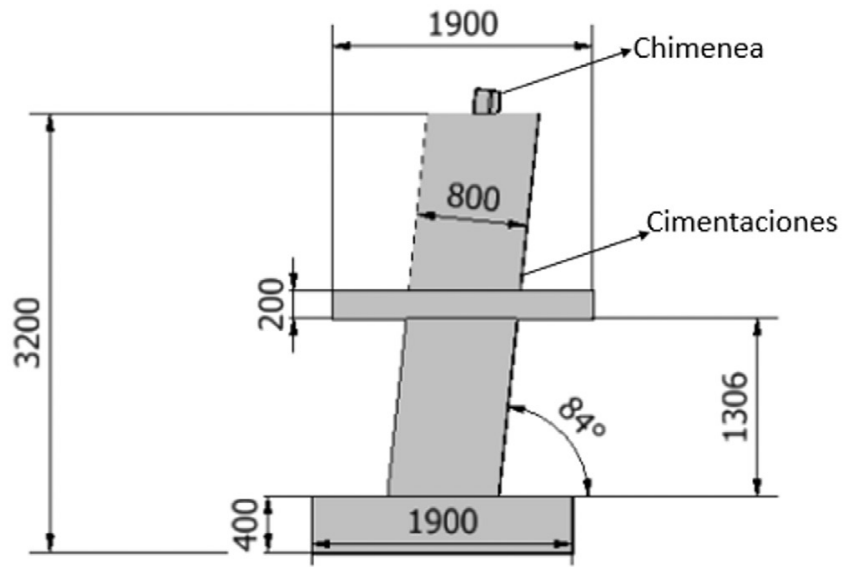

Figura 3. Geometría de la cimentación de zapata doble (unidades en milímetros)
Para el análisis estático se plantea el modelo físico representado en la Figura 4. Se observa que las regiones "bloqueadas" (líneas rojas) en el modelo son las caras superiores de las zapatas, las cuales tienen restricción de movimiento por la masa de tierra sobre ellas, a su vez se desprecian las demás interacciones de la estructura con la tierra, esto es debido a que la deformación que estas pueden llegar a sufrir es mínima.

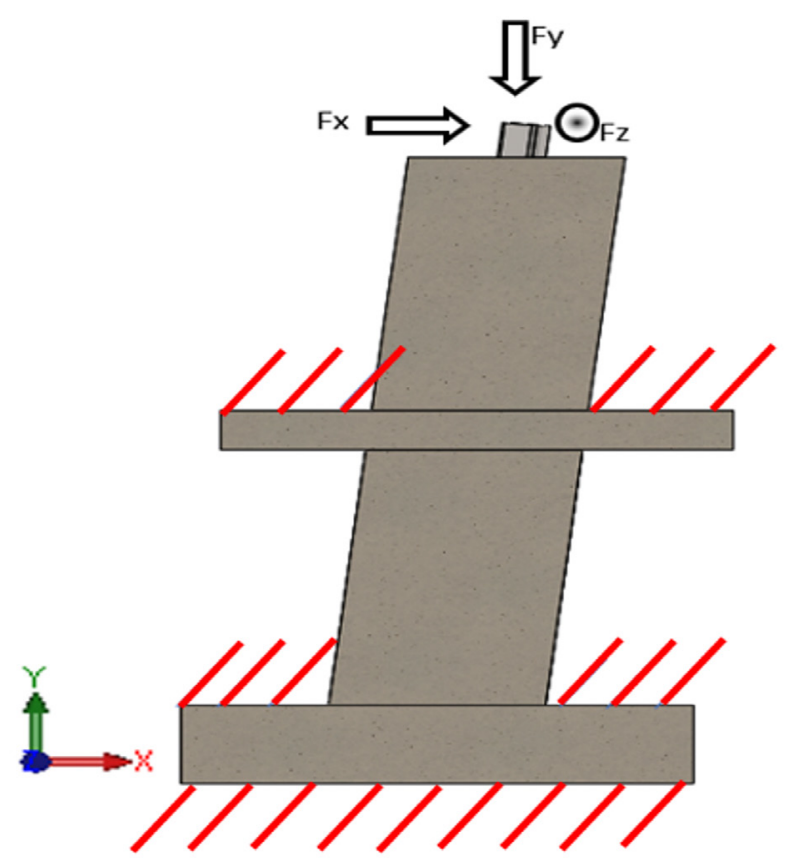

Figura 4. Modelo físico

El análisis FEM fue realizado con base al modelo físico considerando 3 dimensiones. Dentro del modelo computacional se tomaron en cuenta las siguientes consideraciones:

- El material es isotrópico lineal.

- El análisis es estático lineal.

- La estructura recibe cargas de viento de manera perpendicular a los cables, por lo cual se determina solo una componente de acción. También se consideran que las fuerzas de viento actúan de forma puntual y se transforman en cargas torsionales en el punto de estudio. [11]

- Se considera una distribución uniforme en los puntos de apoyo de la torre de transmisión, por ende, las cargas asociadas se dividen en cuatro para realizar el análisis.

- Se considera Acero ASTM A36 estructural para la chimenea en el cual va soportada la estructura; junto con hormigón armado.

- Para el hormigón, dado que es un material frágil se utiliza el esfuerzo último (ver Tabla 1) [12].

- Para el acero debido a que es un material dúctil se utiliza el esfuerzo de fluencia (ver Tabla 1) [12]. 
- Sobre la chimenea se considera una carga distribuida en su región sobresaliente, correspondiente a la carga efectuada por la torre de transmisión.

En la Tabla 1 se observan las características principales de los dos materiales de estudio tomados de la base de datos de ANSYS, Esfuerzo de fluencia a tracción y esfuerzo último a tracción (EFUT), Esfuerzo de fluencia a compresión y esfuerzo último a compresión (EFUC) y junto con la densidad del material.

Tabla 1. Propiedades de los materiales de estudio

\begin{tabular}{llll} 
Material & $\begin{array}{l}\text { EFUT } \\
(\mathrm{MPa})\end{array}$ & $\begin{array}{l}\text { EFUC } \\
(\mathrm{MPa})\end{array}$ & $\begin{array}{l}\text { Densidad } \\
\left(\mathrm{kg} / \mathrm{m}^{3}\right)\end{array}$ \\
\hline $\begin{array}{l}\text { Acero } \\
\text { estructural }\end{array}$ & 250 & 250 & 7850 \\
Hormigón & 5 & 41 & 2300 \\
\hline
\end{tabular}

Fuente: [13 - 14]

Las cargas utilizadas para la evaluación corresponden a las establecidas en un caso de estudio tomado de la literatura [11]. Los valores específicos se encuentran en la Tabla 2; en ésta se resumen las fuerzas en las direcciones X, Y y Z, para las cargas generadas por las corrientes de viento, las cuales se consideran que están siendo aplicadas en la región mostrada en la Figura 5, esto con la intención de asemejar las condiciones de conexión de la chimenea con el resto de la torre de transmisión.

Para confirmar que los valores entregados por la simulación en el programa ANSYS son correctos se utiliza la curva de convergencia mostrada en la Figura 6, donde se comparan el número de elementos del modelo con respecto al esfuerzo máximo de Von Mises. Este criterio va a utilizarse para determinar si existe falla o no en el sistema y cuáles son las dimensiones con las que la cimentación de zapata doble soportaría las cargas asociadas a la torre de transmisión como si estuviera soportada en una cimentación de zapata simple.

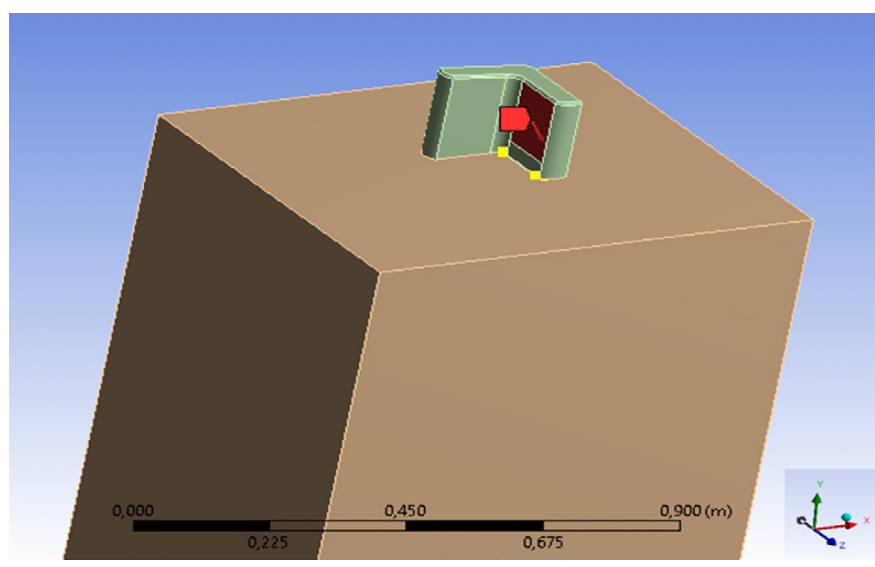

Figura 5. Enfoque que muestra las cargas aplicadas
Las cargas utilizadas para la evaluación corresponden a las establecidas en un caso de estudio tomado de la literatura [11]. Los valores específicos se encuentran en la Tabla 2; en ésta se resumen las fuerzas en las direcciones X, Y y Z, para las cargas generadas por las corrientes de viento, las cuales se consideran que están siendo aplicadas en la región mostrada en la Figura 5, esto con la intención de asemejar las condiciones de conexión de la chimenea con el resto de la torre de transmisión.

Para confirmar que los valores entregados por la simulación en el programa ANSYS son correctos se utiliza la curva de convergencia mostrada en la Figura 6, donde se comparan el número de elementos del modelo con respecto al esfuerzo máximo de Von Mises. Este criterio va a utilizarse para determinar si existe falla o no en el sistema y cuáles son las dimensiones con las que la cimentación de zapata doble soportaría las cargas asociadas a la torre de transmisión como si estuviera soportada en una cimentación de zapata simple.

Tabla 2. Cargas usadas en el modelo.

\begin{tabular}{llll}
\hline $\begin{array}{l}\text { Ángulo } \\
\text { del } \\
\text { viento }\end{array}$ & $\begin{array}{l}\mathrm{X}(\mathrm{N}) \\
\text { Transversal }\end{array}$ & $\begin{array}{l}\mathrm{Y}(\mathrm{N}) \\
\text { Vertical }\end{array}$ & $\begin{array}{l}\mathrm{Z}(\mathrm{N}) \\
\text { Longitudinal }\end{array}$ \\
\hline $0^{\circ}$ & 126950 & 273185 & 0 \\
$30^{\circ}$ & 109942 & 273185 & 75984 \\
\hline
\end{tabular}

Fuente: [11]

El análisis de convergencia se analiza en la Figura 6 donde se observa que el modelo se estabiliza en un punto cercano a los 20.000 elementos, donde la variación del valor máximo del esfuerzo de von mises es baja. Para una mayor fiabilidad y para compensar el tiempo computacional se realiza un análisis con una malla de 30.101 elementos.

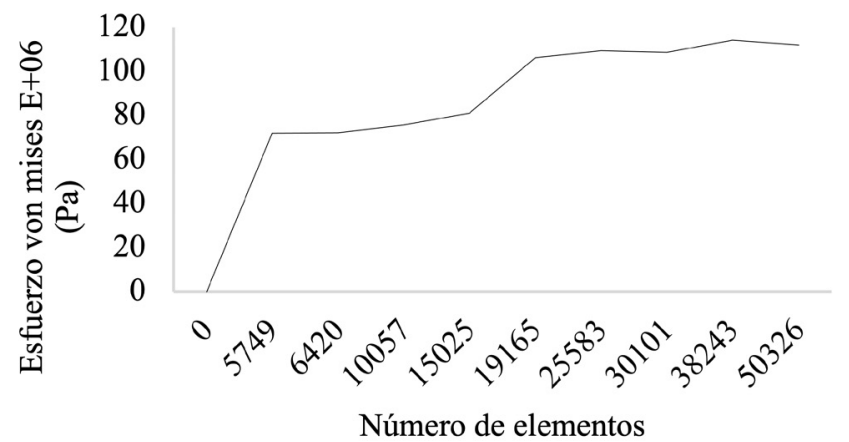

Figura 6. Curva de convergencia (esfuerzos en Pa). 


\section{Análisis de resultados}

Los resultados obtenidos de la simulación numérica en elementos finitos, usando ANSYS versión 20 R2, para el primer análisis indicado en la tabla 2 , ángulo del viento $=$ $0^{\circ}$, es decir en dirección perpendicular a la dirección de los cables de tensión (ubicada sobre el eje Transversal X). La Figura 7 hace referencia a los esfuerzos por Von Mises, y es de destacar que la mayor concentración de esfuerzos se encuentra en la cara en la que están aplicadas las fuerzas en la chimenea de la estructura. Este resultado es congruente con la realidad, debido a que se está considerando que las superficies superiores de las zapatas están bloqueadas con la tierra ubicada encima de las mismas.

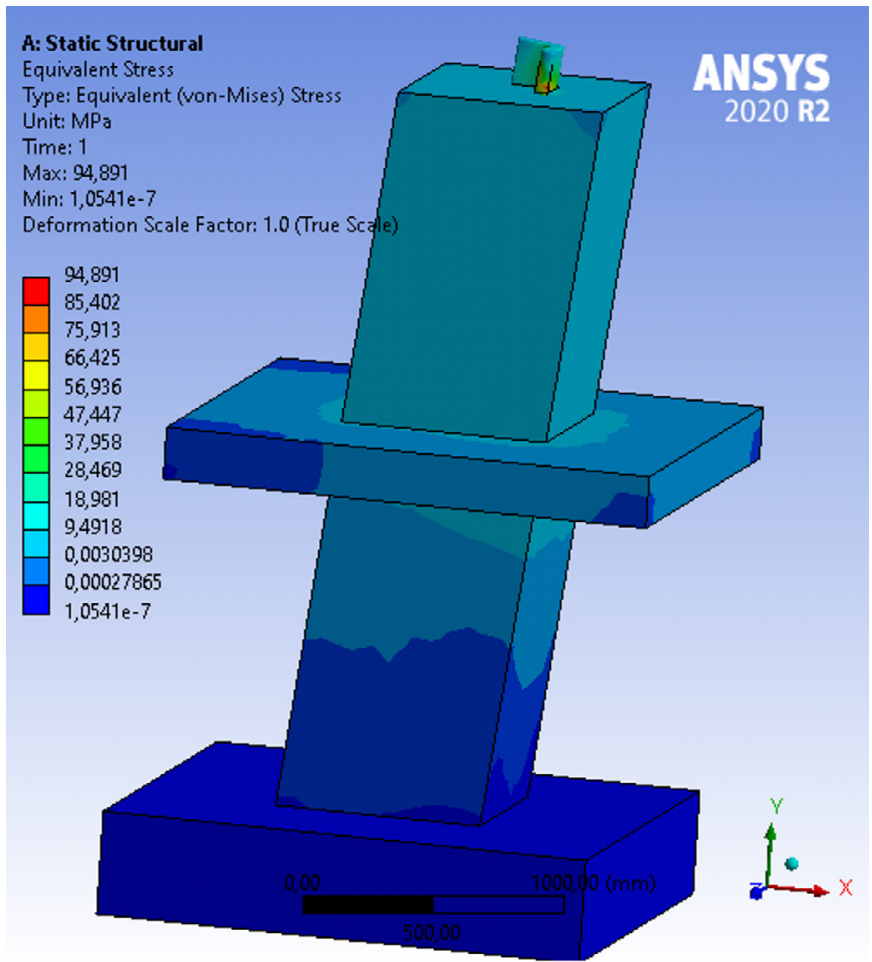

Figura 7. Esfuerzos de Von Mises (Pa) en el modelo a $0^{\circ}$

Se observa que la concentración de esfuerzos es bastante alta en la región donde el acero sobresale del hormigón; por lo cual en la Figura 8 se hará un acercamiento de esta, con la intención de observar las regiones de mayor solicitación.

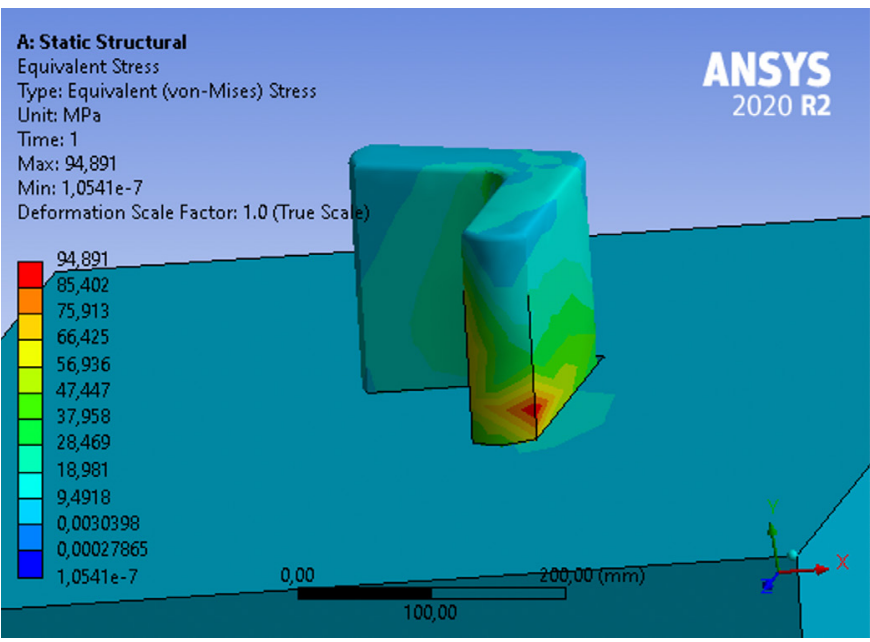

Figura 8. Enfoque esfuerzos Von Mises (Pa) en el modelo a $0^{\circ}$

Las deformaciones en el modelo se observan en la Figura 9, se destaca que la máxima deformación observada es de $0.35 \mathrm{~mm}$.

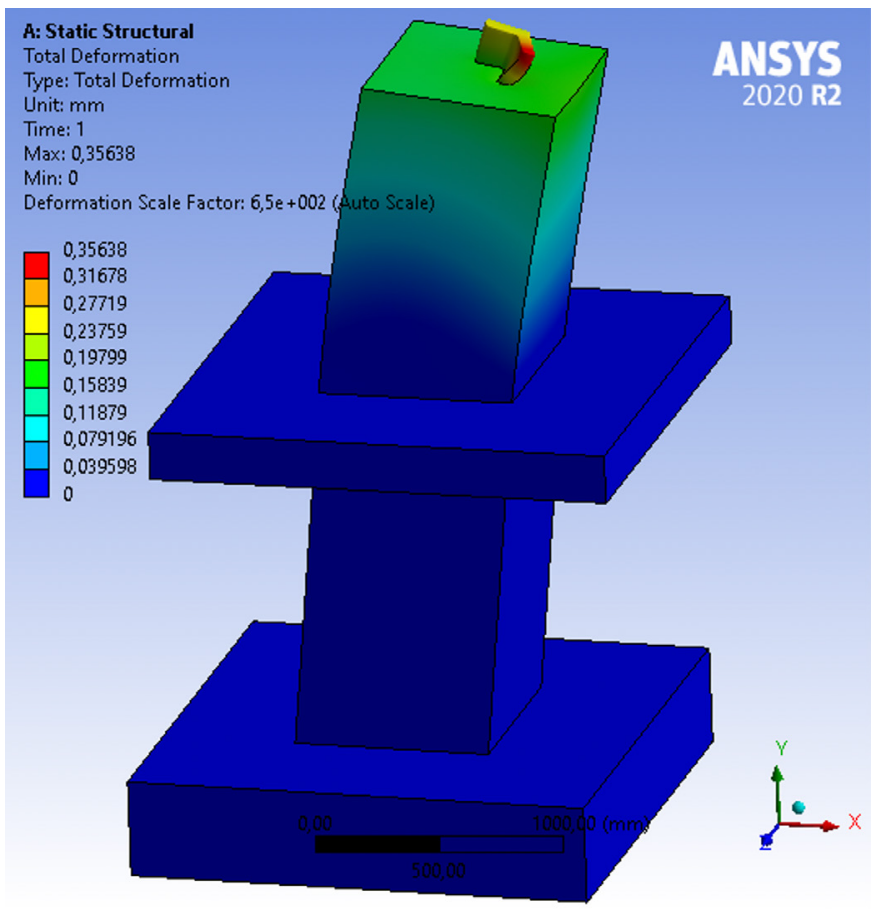

Figura 9. Deformaciones $(\mathrm{mm})$ en el modelo a $0^{\circ}$

Se realiza un acercamiento de la región expuesta a las máximas deformaciones para mostrar la distribución de estas en la estructura. En la Figura 10 se observa el acercamiento propuesto. 


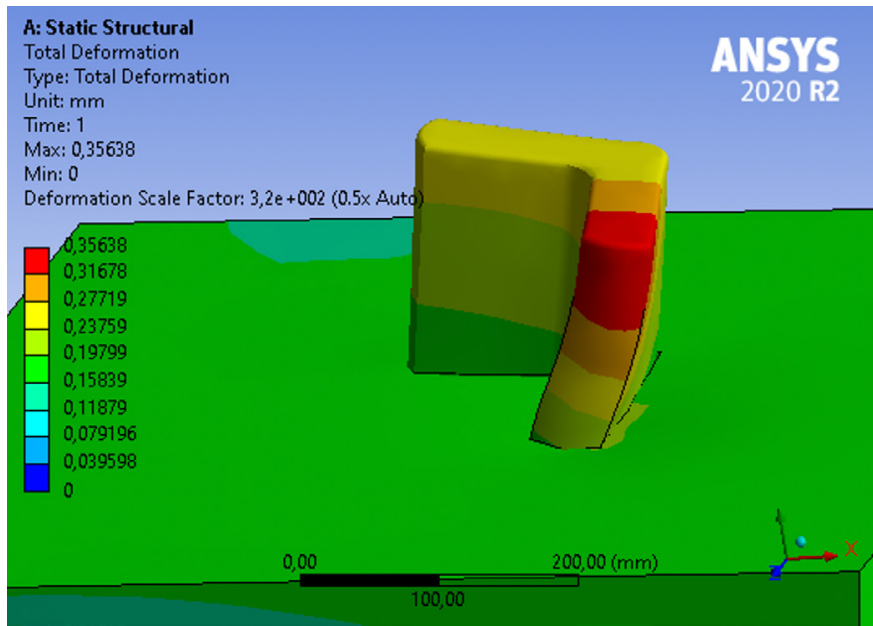

Figura 10. Enfoque deformaciones $(\mathrm{mm})$ en el modelo a $0^{\circ}$

En la Figura 11 se observan los resultados cuando se utilizan las cargas asociadas al ángulo de incidencia del viento de $30^{\circ}$, en este se observa que las cargas están ubicadas, tanto en el eje transversal X, como longitudinal Z.

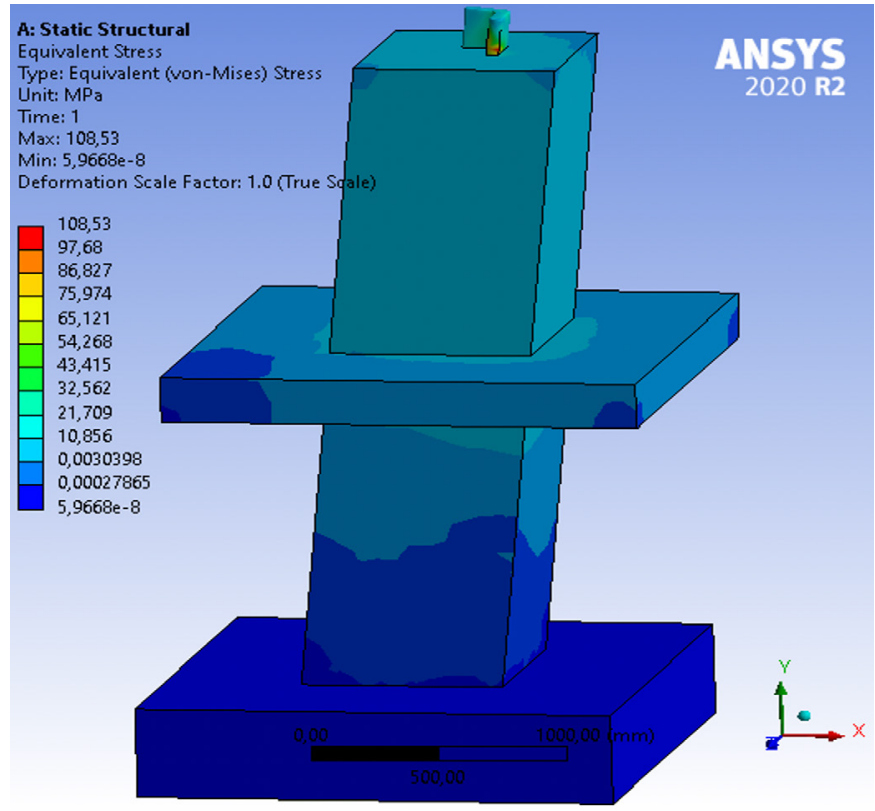

Figura 11. Esfuerzos Von Mise (Pa) en el modelo $30^{\circ}$

La región de mayor solicitación se encuentra en donde el acero sobresale del hormigón, por lo cual se le realiza un acercamiento en la Figura 12 para observar con mayor precisión la distribución de cargas; el hormigón encuentra a $21 \mathrm{MPa}$, mientras que el acero, alcanza el valor máximo de $108 \mathrm{MPa}$.

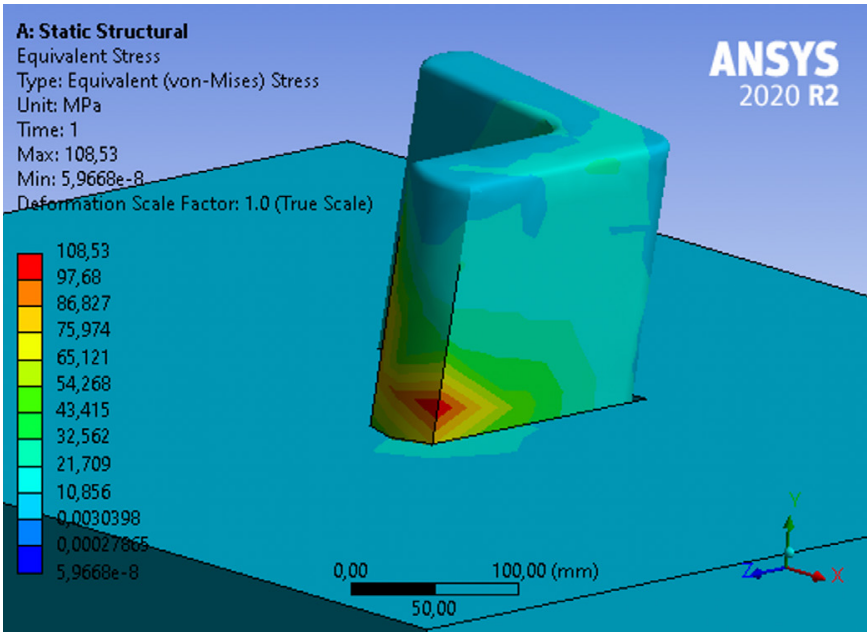

Figura 12. Enfoque esfuerzos Von Mises $(\mathrm{Pa})$ en el modelo a $30^{\circ}$

Las deformaciones observadas en la Figura 13 tienen un valor máximo de $0.36 \mathrm{~mm}$, lo cual es despreciable dado que está varios ordenes de magnitud debajo con respecto a la geometría. A su vez se observa que la chimenea es la que mayor deformación sufre.

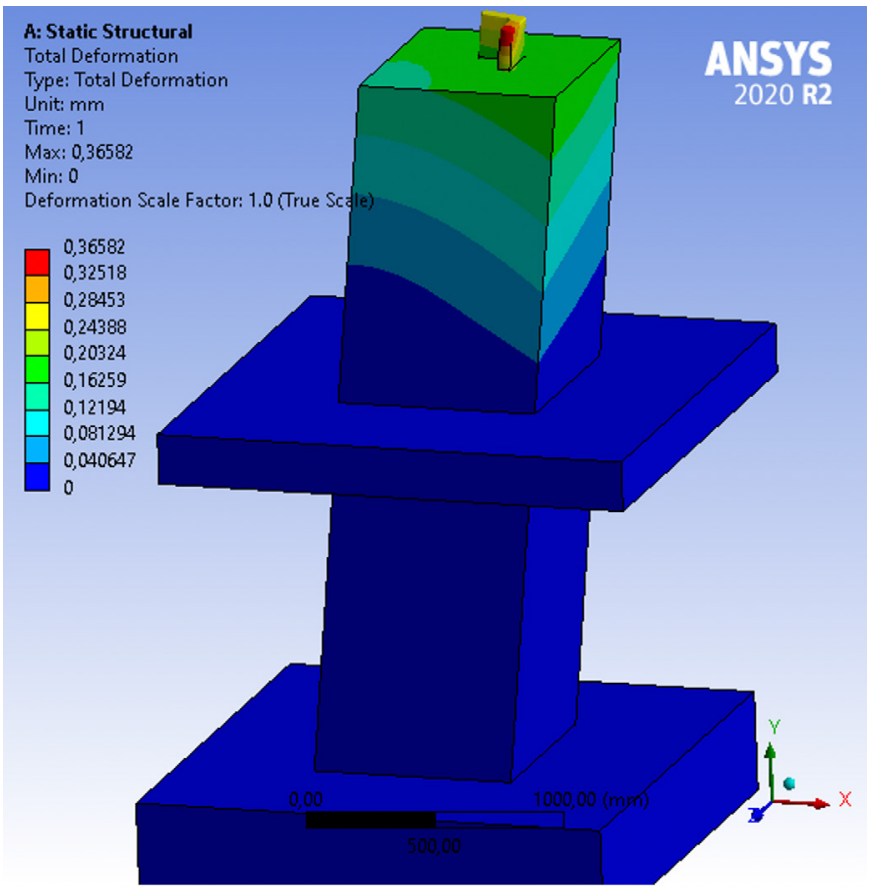

Figura 13. Deformaciones ( $\mathrm{mm}$ ) en el modelo a $30^{\circ}$

Se enfoca en la región visible de la chimenea y se presenta en la Figura 14, para observar la distribución de deformaciones a las que está sufriendo el sistema. 


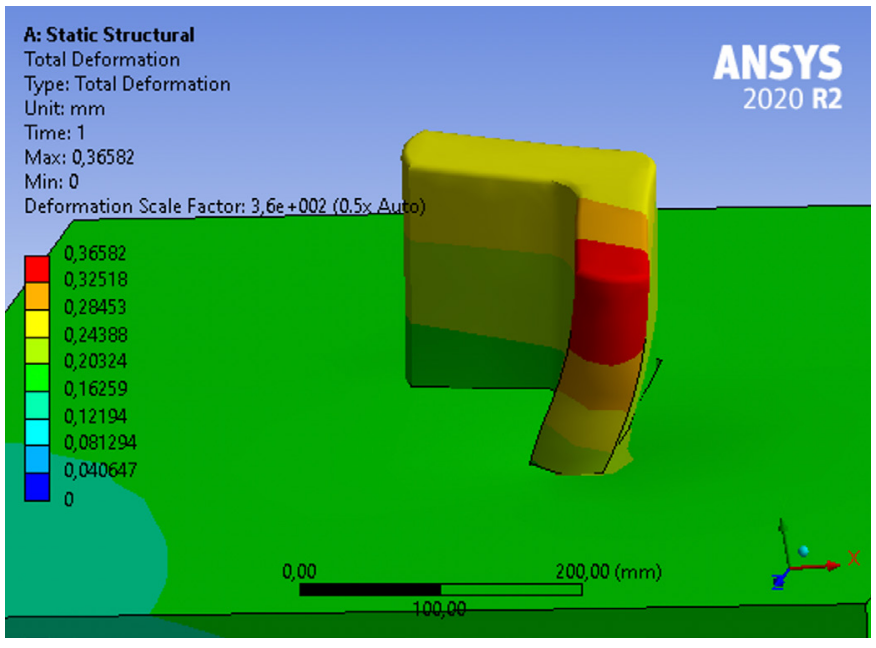

Figura 14. Enfoque deformaciones $(\mathrm{mm})$ en el modelo a $30^{\circ}$

En base a estos resultados se compararán los valores máximos con el máximo soportado. En la Tabla 3 se presenta esta comparación, donde el esfuerzo máximo (EM) hallado en la simulación se compara con el esfuerzo máximo soportado (EMS) para cada material según las hipótesis planteadas anteriormente [13-14].

Tabla 3. Esfuerzos y factores de seguridad (FS)

\begin{tabular}{ccccc}
\hline Material & $\begin{array}{c}\text { Ángulo } \\
\left({ }^{\circ}\right)\end{array}$ & $\begin{array}{c}\text { EM } \\
(\mathrm{MPa})\end{array}$ & $\begin{array}{c}\text { EMS } \\
(\mathrm{MPa})\end{array}$ & FS \\
\hline $\begin{array}{c}\text { Acero } \\
\text { estructural }\end{array}$ & 0 & 94.89 & & \\
& 30 & 108.53 & 250 & 2.30 \\
Hormigón & 0 & 15.45 & 41 & 2.51 \\
& 30 & 16.33 & & \\
\hline
\end{tabular}

Se realiza una comparación con la zapata simple cuyas dimensiones se observan en la Figura 15. En la Tabla 4 se presenta un comparativo entre los volúmenes de zapata simple y zapata doble, tanto de excavación como de material.

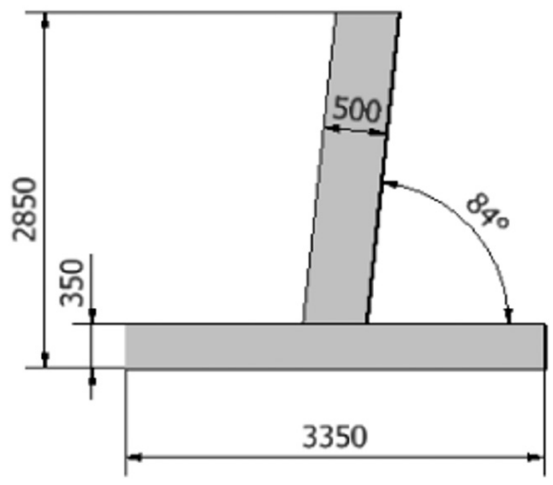

Figura 15. Cimentación de zapata simple de referencia (unidades en $\mathrm{mm}$ ) [6]
La disminución del volumen de material es de $0.75 \mathrm{~m} 3$, de esta forma la cimentación de zapata doble representa el $83.5 \%$ del volumen total de la cimentación por zapata simple, ahorrando casi un $17 \%$ de material; a su vez la diferencia en la excavación es de $19.18 \mathrm{~m} 3$, lo cual implica que para la construcción de la zapata doble podría llevar a una reducción del $60 \%$ del volumen excavado. Adicionalmente esta reducción representa una disminución en diversos factores, como lo es el tiempo de transporte, excavación y construcción, dicho esto, es evidente la reducción en el costo del material utilizado.

Tabla 4. Comparativa de volúmenes aproximados de concreto zapata simple y zapata doble en $\mathrm{m} 3$.

\begin{tabular}{lll}
\hline & Zapata simple & Zapata doble \\
\hline Excavación & 31.98 & 12.80 \\
Material & 4.55 & 3.83 \\
\hline
\end{tabular}

\section{Conclusiones}

Según el modelo planteado la geometría propuesta logra soportar las condiciones de carga sin fallar ni sufrir grandes deformaciones; esto confirma que estáticamente la estructura funciona.

El criterio de falla de Von Mises nos confirma que tanto el acero estructural utilizado para la chimenea, como el hormigón funcionan a la perfección para las condiciones de carga dadas. Se observa que el esfuerzo máximo de tracción del acero es de $250 \mathrm{MPa}$ y el esfuerzo máximo al que es sometido es de $108.53 \mathrm{MPa}$; esto nos dice que no falla y tiene un factor de seguridad de 2.30. Así mismo el hormigón utilizado soporta como máximo $41 \mathrm{MPa}$ y está sometido a 16.33 $\mathrm{MPa}$, teniendo como factor de seguridad 2.51.

Como estudio futuro se recomienda realizar un análisis de la construcción por bloques prefabricados, dado que estos son un factor importante para la fabricación de la geometría planteada. Los factores de seguridad encontrados son de 2.30 y 2.51 para el acero y el hormigón respectivamente, aunque son inferiores al valor recomendado en la literatura $(\mathrm{FS}=3)$ [15] se tiene que considerar que es una geometría todavía en estudio, la cual no está restringida para cambios en sus dimensiones y análisis de optimización, los cuales podrían aumentar el factor de seguridad de la estructura.

El trabajo realizado se basa solo en el estudio estático de la estructura; para asegurar sus condiciones de operación y determinar si el diseño planteado puede llegar a reemplazar la geometría actualmente utilizada se requiere realizar un análisis de estabilidad al volumen de tierra asociado al sistema, para confirmar el comportamiento que este tiene y si puede aportar la rigidez necesaria para que la geometría planteada pueda funcionar. 
La geometría planteada al igual que la geometría convencional dependen directamente de las condiciones del suelo sobre el cual van a ser construidas, por lo cual las geometrías utilizadas en este trabajo son solo representativas y se recomienda que de ser utilizadas se determinen las condiciones del lugar a construir y se realice un correcto estudio de sus implicaciones sobre las geometrías.

\section{Referencias}

[1] A. Sarabia-Guarin, J. Sánchez-Molina y

J.C. Leyva-Díaz "Uso de nutrientes tecnológicos como materia prima en la fabricación de materiales de construcción en el paradigma de la economía circular", Respuestas, vol. 22, no. 1, pp. 6-16, 2017. Doi: https://doi. org/10.22463/0122820X.815

[2] I. N. Coronilla Osorio. "Estudio de la mejora de terreno mediante columnas suelo-cemento tipo Mixpil". Tesis doctoral. Universidad de malaga. 2017.

[3] Aramayo A., Amayo M., Paniagua D., "Hormigón con Nanotecnología", Estudiantes $\mathrm{N}^{\circ}$ 692, Facultad de ciencias tecnológicas, y Pontificia de San Francisco Xavier de Chuquisaca, Bolivia, 2014.

[4] J. W. Mayta Rojas. "Influencia del aditivo superplastificante en el tiempo de fraguado, trabajabilidad y resistencia mecánica del concreto en la ciudad de Huancayo". Tesis de pregrado.Universidad nacional del centro del Perú. 2014.

[5] F. Delgado Ramos. "Sistema de cimentación modular de instalación y desinstalación rápida para la construcción de presas hidráulicas inflables y presa hidráulica que lo contiene”. Oficina de patentes España ,Patente 2386607.,2013.

[6] V. Guerrero Flores, N. Melchor García, "Normalización de cimentaciones superficiales para torres de transmisión",

in XII congreso nacional de ingeniería estructural, Morelia, Michoacán, México, 1999, p. 14.

[7] S. K. Satish Kumar, 8.5 "Structural arrangement of foundation". Indian institute of technology. 2015.

[8] E. Juarez Badillo, A. Rico Rodríguez. Mecánica de suelos Tomo II. México DF. Editorial Limusa. 1973

[9] J. I. de Llorens Duran and E. Pujadas Gispert, “Cimentaciones prefabricadas para módulos de vivienda 3D" in V Congr. ACHE, Escuela Técnica Superior de Arquitectura de Barcelona de la Universidad Politécnica de Cataluña 2011,p. 10.

[10] D. Gaspar-Tebar. "Normalización del cemento. Tiempo de fraguado:algunos comentarios sobre el método de ensayo". Materiales de con- strucción, vol 30, no 178. 1980. Doi: https://doi. org/10.3989/mc.1980.v30.i178.1078

[11] C. Alzate Acevedo, "Diseño Conceptual de torres de trasmisión de energía utilizando el método de optimización topológica considerando Análisis tridimensional y cargas producidas por cables y vientos", Tesis de Maestría, Universidad Nacional de Colombia , 2016,pp.189.

[12] R. C. Hibbeler. Mecánica de materiales. 8va edición. Naucalpan de Juarez. Pearson. 2011

[13] J. MattheiB, Hormigón. Barcelona: Ed. Revertté, S.A., (1980).

[14] Astm a 36/a 36M-05, "Standard Specification for Carbon Structural Steel" Standards, vol. 14, pp. 1-4, 2015. Recuperado de: https://www.academia.edu/35304475/Standard_Specification_for_ Carbon Structural Steel 1

[15] "TEMĀ4. CIMENTACIŌNES SUPERFICIALES”, Ingeniería geotécnica, departamento de arquitectura, urbanismo y construcción, Universidad Politécnica de Cataluña, 2015, https://portal. camins.upc.edu/materials_guia/250240/2012/ tema_4_ciment_super.pdf 